\title{
Hemocompatibility research on the micro-structure surface of a bionic heart valve
}

\author{
Xia Ye ${ }^{\mathrm{a}, *}$, Ze Wang ${ }^{\mathrm{a}}$, Xianghua Zhang $^{\mathrm{a}}$, Ming Zhou ${ }^{\mathrm{b}}$ and Lan Cai ${ }^{\mathrm{b}}$ \\ ${ }^{a}$ School of Mechanical Engineering, Jiangsu University of Technology, Changzhou, Jiangsu 213001, \\ China \\ ${ }^{b}$ Center of Photonics Fabrication, Jiangsu University, Zhenjiang, Jiangsu 212013, China
}

\begin{abstract}
In order to study how the geometric parameters and shape of the micro-structure surface of a bionic heart valve affects hemocompatibility, mastoid micro-structures with different periodic space were fabricated using a femtosecond laser on a polyurethane (PU) surface. The apparent contact angles of droplets on the micro-structure surfaces were measured to characterize their wettability. Then a series of blood compatibility experiments, including platelet adhesion, dynamic coagulation and hemolysis were completed. The experimental results showed that the micr-ostructure on the biomaterial surface helped improve its hydrophobicity and hemocompatibility. Also, the periodic space affected not only the hydrophobicity but also the hemocompatibility of the biomaterial. With the increasing of the periodic space, the apparent contact angle increased, the number of platelet adhesion decreased, the dynamic clotting time became longer and the hemolysis ratio reduced. In addition, the shape of the micro-structure also affected the hemocompatibility of the biomaterial.
\end{abstract}

Keywords: Hemocompatibility, micro-structure surface, polyurethane, periodic space, bionic heart valve

\section{Introduction}

Artificial heart valve replacement is one of the most effective and lasting ways to save the life of a patient whose heart valve underwent serious pathological changes. In recent decades, many researches about the artificial mechanical heart valve have been focused on finding new materials and modifying valve surfaces. Therefore, valve research is developing very fast and some notable progresses have been made. However, bio-compatibility, especially the hemocompatibility of emerging materials, is still a major bottleneck that hinders the development of artificial heart valves. In general, after artificial heart valve implantations, blood components (such as protein, fat or calcium) can be absorbed or deposit to the vavle surface due to its long-term presence, and precipitation and adsorption of platelets and coagulation would occur. Clotting and thrombus formation are among the most important factors that impair normal valve function, and even cause failure of the implantation. Therefore, the solution to improve the hemocompatibility of mechanical valves is currently the

\footnotetext{
${ }^{*}$ Corresponding author: Xia Ye, School of Mechanical Engineering, Jiangsu University of Technology, Changzhou, Jiangsu 213001, China. Tel.:13915052185; Fax: +86-0519-86953200; E-mail: yexia@jsut.edu.cn.
} 
emphasis of related researches. It not only has important scientific significance, but also has great economic and social values.

So far, studies on the artificial valve hemocompatibility mainly focused on surface modifications such as surface coating or film covering [1,2]. All et al. [3] deposited Cr-modified diamond-like carbon (Cr-DLC) films onto silicon wafers using a magnetron sputtering process. Leng et al. [4] studied the blood compatibility of titanium alloy artificial heart valves by using $\mathrm{TiO}_{2} / \mathrm{TiN}$ duplex films, and their result showed that the titanium oxide improves blood compatibility and the TiN film mainly improves the mechanical properties of the films. Bolz et al. [5] deposited a coating of amorphous hydrogenated silicon carbide (a-SiC:H) using plasma-enhanced chemical vapor deposition (PECVD). Krzysztofet et al. [6] coated nanocrystalline diamond (NCD) onto the titanium alloy $\mathrm{Ti}_{6} \mathrm{Al}_{4} \mathrm{~V}$ with the use of the RF PACVD technique. J.M. Hung et al. [7] also studied Nanocrystalline diamond (NCD) films on a silicon substrate by using a microwave plasma chemical vapor deposition procedure with $\mathrm{CH}_{4} / \mathrm{Ar} / \mathrm{H}_{2}$ gas mixture. Zhang et al. [8] deposited a silicon-alloy pyrolytic carbon coatings consisted of pyrolytic carbon and $\beta$ silicon carbides on the mechanical heart valve's surface.

Although all researches showed that the hemocompatibility of artificial valves could be improved by a variety of treatments on its surface, they are still less than satisfactory. Since the surfaces of artificial valves are mostly curved, it is very hard to guarantee the homogeneity of the coating or film on it. In addition, cohesion between the coating and the base material of the artificial valve also largely affects its durability.

In our previous study on animals' heart valves, we found that micro-nano composite hierarchical structure on the heart valve surface plays a very important role in good blood compatibility [9]. At the same time, there were lots of researches trying to improve the properties of biomaterials by constructing micro-structure on the material surface. These interested properties include superhydrophobicity, low adhesion, reduced drag, oleophobicity and wear-resistance [10-15]. In this paper, micro-structures similar to that found on the animal heart valve surface were fabricated using femtosecond laser technology on one of the biological materials-polyurethane (PU) surface. Then the effect of this fine structure on the hemocompatibility was studied through a platelet adhesion experiment, dynamic coagulation and hemolysis test experiment.

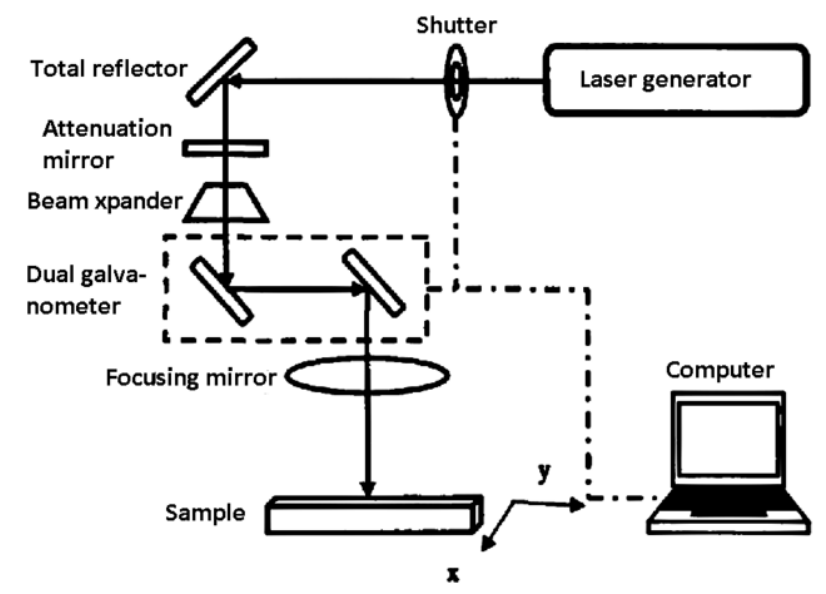

Fig. 1. Diagrammatic sketch of femtosecond laser etching device to manufacture the micro-structure. 


\section{Experimental method}

\subsection{Preparation and characterization of sample}

Periodic micro-structures were fabricated using a femtosecond laser processing system as shown in Figure 1. The laser source was a Femtosecond Regenerative/Multi-pass Ti: Sapphire Amplifier with $120 \mathrm{fs}$ pulse width, $800 \mathrm{~nm}$ center wavelength and $1 \mathrm{KHz}$ repetition rate. The laser pulse energy can be adjusted by a power meter. The energy range was $1-2.5 \mathrm{~mJ}$. The entire preparation process was in the atmosphere environment at $20^{\circ} \mathrm{C}$.

To construct the periodic mastoid micro-structure, a laser was scanned on the PU surface in two mutually perpendicular directions successively with different pulse energy (in the direction $\mathrm{X}$ is 40 $\mathrm{mW}$ and in the direction $\mathrm{Y}$ is $20 \mathrm{~mW}$ ). In order to obtain the different periodic space of the mastoid micro-structure on the PU surface, different scanning space was selected in the processing, which was $20,30,40,50 \mu \mathrm{m}$ respectively.

The processed samples were placed in an ultrasonic cleaning machine, washed with deionized water at room temperature for 30 minutes, rinsed 3 times with deionized water, dried with cold air, and then treated by $\mathrm{SiH}_{4}$ in a vacuum oven for $3 \mathrm{~h}$ at $90^{\circ} \mathrm{C}$. The samples in the oven were incubated for $1 \mathrm{~h}$ and taken out when they were cold.

The apparent contact angle (CA) on the samples surfaces were measured by a video-frequency optics goniometer (OCAH200, Dataphysics) using the sessile drop method. The volume of the deionized water drop used in the test was $1 \mu \mathrm{L}$. In order to reduce the measurement error, five different points were measured on each sample surface, and the average of the five measurements was taken as the final measurement result. In order to compare the hydrophobicity of the micro-structure surface with that of a flat surface, the CA of the water on the smooth PU surface was also measured.

\subsection{Blood compatibility test}

There are three main methods to evaluate the blood compatibility of biomaterials, the vitro method, semi-vivo method and vivo method. In this paper, the in vitro method was adopted. Briefly, blood from the body was applied on the test material. Then the short-term effects of blood proteins, red blood cells, and platelets on the biomaterial surface were observed and tested. Although measurement error is larger than that of other methods, the in vitro method is simple, fast and economical. It is suitable for making comparisons among the same set of experimental materials and initial screening of biological materials.

\subsubsection{Platelet adhesion}

Micro-structured PU surfaces with different periodic space were cleaned in the Ultrasonic Cleaner with deionized water for three times and then soaked for two hours in phosphate buffer solution (PBS, $\mathrm{pH}$ 7.4). At the same time, fresh human blood added with anticoagulant citrate dextrose (9:1) was centrifuged at $1000 \mathrm{rpm}$ for $10 \mathrm{~min}$ to obtain platelet-rich plasma (PRP). Then samples were incubated in PRP in a constant-temperature bath at $37^{\circ} \mathrm{C}$ for $30 \mathrm{~min}$. After being cleaned three times with PBS to remove non-adherent platelets, the samples were equilibrated in $2.5 \mathrm{vol} \%$ glutaraldehyde for two hours to fix the adhered platelets. Then they were rinsed with PBS ( $\mathrm{pH} 7.4$ ) again, and dehydrated in a series of gradual ethanol/distilled water mixtures from $30 \%$ to $100 \%$ ( $20 \%$ ethanol increment) for 30 min each. After drying at room temperature, the samples surfaces were treated by spray-gold using the magnetron sputtering technique and tested by Scanning Electron Microscope (SEM). The results of 
SEM experiments were shown with representative micrographs. Eight to ten fields of view were chosen randomly to obtain good statistical density of the platelet adhesion. The final number of platelets adhering to the surface was expressed as the percentage of the control.

\subsubsection{Dynamic coagulation}

Every test sample was placed in the center of a small beaker bottom which stayed at a constant temperature of $37^{\circ} \mathrm{C}$ for $5 \mathrm{~min}$. Then $0.25 \mathrm{ml}$ of fresh blood was injected into the bottom of the beaker using a micropipette. The beaker stayed at a constant temperature of $37^{\circ} \mathrm{C}$ for $5 \mathrm{~min}$ again, then 0.02 $\mathrm{ml}$ of $\mathrm{CaCl}_{2}$ solution $(0.2 \mathrm{~mol} / \mathrm{L})$ was injected in the blood and gently stirred well. After the droplet stayed for a certain time (six time points were adopted in this experiment: 10, 20, 30, 40, 50, 60 min respectively), $25 \mathrm{ml}$ of distilled water was added to the beaker. Then the red blood cells which were not solidified on the sample surface underwent a hemolytic reaction, and the free hemoglobin would uniformly disperse in the water. The concentration of hemoglobin in the water was characterized by absorbance measurements using a continuous wavelength microplate reader (wavelength $540 \mathrm{~nm}$ ).

\subsubsection{Hemolysis test}

In order to be for future use, $4 \mathrm{ml}$ of fresh human blood added with anticoagulant citrate dextrose was diluted with $5 \mathrm{ml}$ of $0.9 \% \mathrm{NaCl}$ solution (namely physiological saline). The sample, which was cleaned and dried according to the previous method, was placed in a glass vessel, then $10 \mathrm{ml}$ of physiological saline was added and the vessel stayed in a constant-temperature bath at $37^{\circ} \mathrm{C}$ for $30 \mathrm{~min}$. $0.2 \mathrm{ml}$ of diluted blood was added to the vessel and mixed uniformly. At the same time, in another glass vessel, $10 \mathrm{ml}$ of physiological saline and $0.2 \mathrm{ml}$ of diluted blood were mixed to serve as a negative control. Also, in a third glass vessel, $10 \mathrm{ml}$ of distilled water and $0.2 \mathrm{ml}$ of diluted blood were mixed to serve as a positive control. These three vessels remained in constant-temperature together at $37^{\circ} \mathrm{C}$ for $1 \mathrm{~h}$. Then they were transferred into three microtubes separately, and centrifuged at $1000 \mathrm{rpm}$ for $5 \mathrm{~min}$. The supernatant was pipetted and characterized by absorbance measured using a continuous wavelength microplate reader (wavelength $540 \mathrm{~nm}$ ).

Haemolysis was calculated according to the following equation.

$$
H(\%)=\frac{D_{t}-D_{n e}}{D_{p e}-D_{n e}} \times 100 \%
$$

Where $\mathrm{H}$ is haemolysis, Dt is absorbance values for the experimental samples, Dne is absorbance value for the negative control and Dpe is absorbance value for the positive control.

\section{Results and discussion}

\subsection{Apparent contact angle}

Table 1 shows the apparent contact angles of a droplet on the mastoid micro-structure surfaces. As can be seen from the table, comparing with the smooth PU surface $\left(\theta=110.1^{\circ}\right)$, the superhydrophobicity of all micro-structure surfaces are improved $\left(\theta>150^{\circ}\right)$ because of their micro-structure. X-ray diffraction of the PU surface before and after femtosecond laser processing is 


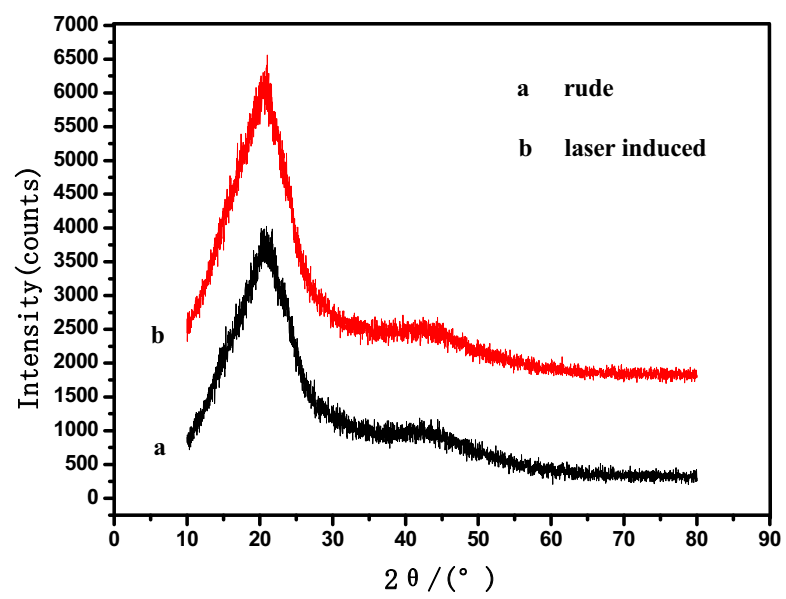

Fig. 2. XRD spectra of the PU surface before and after laser processing.

shown in Figure 2, in which a is the map of the pre-processing and $b$ is the pattern after processing. It can be seen that there was almost no difference between the two spectra, indicating that the processing doesn't change the chemical composition of the PU surface. The wetting properties of a solid surface depend on its surface chemical properties and micro-structure. Therefore, after laser processing, the micro-structure on the PU surface improves the superhydrophobicity. Moreover, the superhydrophobic properties are related to the periodic space of the mastoid micro-structure; the larger the periodic space, the stronger the superhydrophobicity.

\subsection{Platelet adhesion}

Figure 3 shows the adhesion of platelets on the different PU surfaces. It is evident from these images that platelet adhesion to different micro-structure surfaces is not the same. Figure 4 shows the statistical results of the number of platelets that adhered to different surfaces. In this figure, the number of platelets adhering to the micro-structure PU surface was compared with that to the smooth PU surface. It can be seen from the histogram that the number of platelet adhesions decreases with the increase of the period space, which is consistent with the degree of hydrophobic properties of the micro-structure surfaces. However, the case of Figure 3(b) was an exception. Although its surface period space (0.30) is greater than that of Figure 3(a) (0.25), the number of platelet adhesions was greater, which is also consistent with hydrophobic properties (shown in Table 1). The main reason for this exception is that the micro-structure shown in Figure 3(b) was not the ideal mastoid micro-structure, and the apparent contact angle of a droplet on its surface was just $151.1^{\circ}$. Therefore, platelet adhesion to the micro-structure surface relates not only to the size of the micro-structure, but also to its shape.

In addition, while there were some platelets adhering to each mastoid micro-structure PU surface, the adhered platelets showed regular circular shapes that were not deformed. The platelets of this adsorptive form are very easy to return to the bloodstream, so it is a reversible adsorption, and may hardly cause coagulation and thrombosis. 


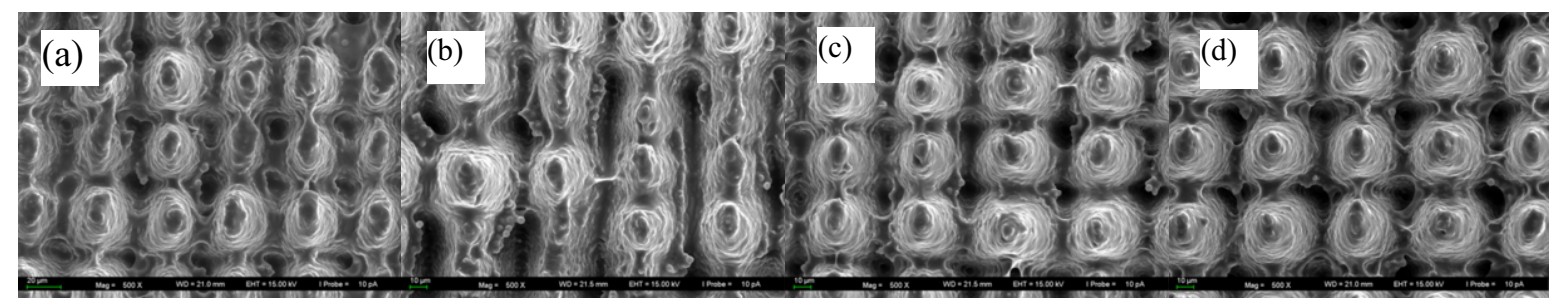

Fig.3. The morphology of platelet adhesion on different PU surfaces. a. Platelet adhension on PU with periodic space of 0.25 . b. Platelet adhension on PU with periodic space of 0.3. c. Platelet adhension on PU with periodic space of 0.43. d. Platelet adhension on PU with periodic space of 0.52 .

\subsection{Dynamic coagulation}

Activation of clotting factors results in the formation of fibrin that then causes coagulation. With different degrees of activation, the clotting time is different. Meanwhile, the degree of the coagulation increases with the prolonging of the time that blood is in contact with the material. For the same period of time, higher absorbance denotes, higher concentration of hemoglobin present in the solution, which also indicates less blood coagulation occurred on the material surface and better blood compatibility. For multiple time durations on the same material, the absorbance curve will reflect the trend of coagulation and the length of clotting time. If the curve of dynamic clotting time is flat, it means that the absorbance value is high, the clotting time is long and the level of blood coagulation factors activated is low. It indicates that the degree of material to cause thrombosis is small and the anticoagulant of the material is good. Therefore, the degree of activation of different micro-structure surface to the blood coagulation factors can be quantitatively compared through the time that is required for blood at different surfaces to achieve the same degree of coagulation. Generally the anticoagulant properties of materials can be indicated through measuring the optical density (OD) of the solution at different contact time points and plotting the curve of OD against time. Usually if the dynamic clotting curve slowly inclines downward and sustains for a very long time, it means that the endogenous factor is activated in a low level and the anticoagulant properties of the materials is excellent. On the contrary, if the dynamic clotting curve rapidly slopes downward and lasts for a short time, it means that the endogenous factor was activated in a high degree and the anticoagulant properties of the materials is not good enough.

Table 1

The apparent contact angle $\theta$ of a droplet on the PU surface with different periodic space

\begin{tabular}{lllllll}
\hline \multirow{2}{*}{ Periodic space } & \multicolumn{2}{c}{ Apparent contact angle } & \multicolumn{3}{l}{} \\
& $\theta_{1}$ & $\theta_{2}$ & $\theta_{3}$ & $\theta_{4}$ & $\theta_{5}$ & $\theta_{v}$ \\
\hline 0.25 & 154.2 & 154.6 & 154.5 & 154.3 & 154.0 & 154.3 \\
0.30 & 151.0 & 151.2 & 150.8 & 151.5 & 151.3 & 151.1 \\
0.43 & 157.1 & 156.8 & 157.0 & 157.3 & 156.9 & 157.0 \\
0.52 & 162.5 & 162.8 & 162.1 & 162.4 & 162.6 & 162.5 \\
\hline
\end{tabular}


Table 2

Kinetic clotting time test for mastoid micro-structure PU surface with different periodic space

\begin{tabular}{lllllll}
\hline Time/min & OD & & & & & \\
periodic space & 10 & 20 & 30 & 40 & 50 & 60 \\
\hline 0.25 & 0.199 & 0.145 & 0.116 & 0.091 & 0.085 & 0.057 \\
0.3 & 0.187 & 0.151 & 0.113 & 0.089 & 0.072 & 0.048 \\
0.43 & 0.205 & 0.157 & 0.12 & 0.102 & 0.094 & 0.059 \\
0.52 & 0.212 & 0.179 & 0.147 & 0.125 & 0.105 & 0.076 \\
Smooth PU & 0.175 & 0.133 & 0.105 & 0.087 & 0.063 & 0.042 \\
\hline
\end{tabular}

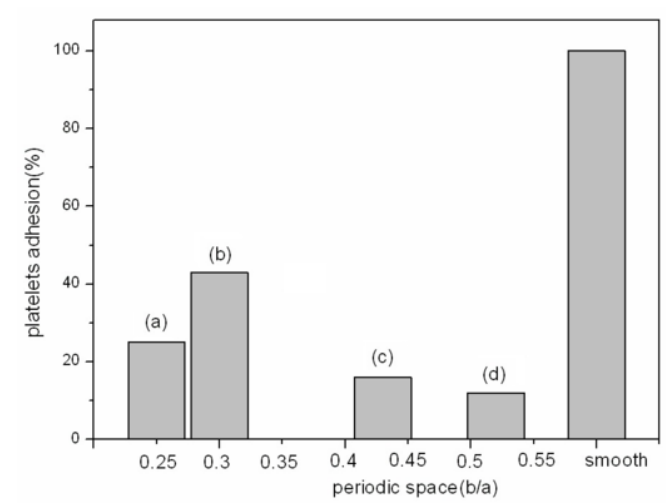

Fig. 4. The statistical number of the platelets adhesion on the different mastoid micro-structure PU surfaces space.

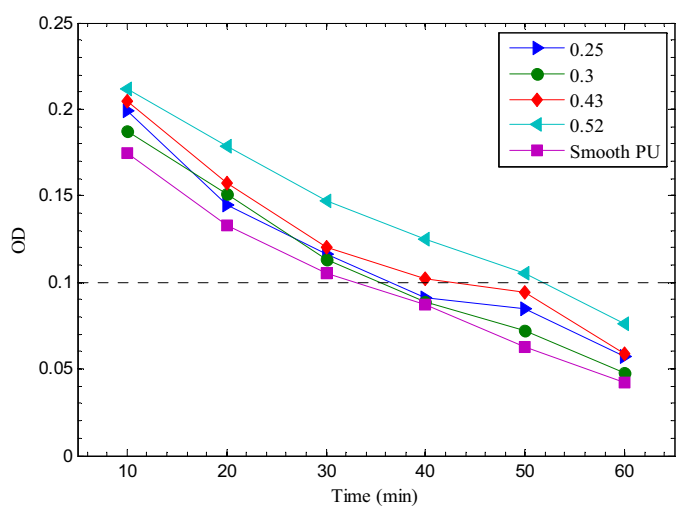

Fig. 5. Kinetic clotting time curves of mastoid micro-structure PU surface with different periodic.

The experimental results of dynamic clotting time on micro-structure PU surfaces with different period space are shown in Table 2. According to Table 2, the dynamic clotting time curve under these serial conditions were drawn (Figure 5). The time when OD equals 0.10 was used as the dynamic clotting time. The dynamic clotting time of the mastoid micro-structure PU surface with the maximum periodic space $(0.52)$ was the longest $(52 \mathrm{~min})$. When the periodic space became smaller $(0.43,0.25)$, its dynamic clotting time correspondingly shortened to $41 \mathrm{~min}$ and $36 \mathrm{~min}$ respectively. However when the periodic space was 0.3 , its dynamic clotting time did not comply with the law, and its time was $35 \mathrm{~min}$, which is shortest. This result is consistent with the results of the platelet adhesion test, in which suboptimal shape of the mastoid micro-structure contributed negatively. In addition, from Figure 5, it can be seen that the dynamic clotting times of all the micro-structure surfaces were longer than that of the smooth PU surface, suggesting that the micro-structure on the surface may help extend the dynamic clotting time and that the shape and period space are all influential factors.

\subsection{Hemolysis test}

When blood contacts the biological material, red blood cells are damaged to different degrees; hemoglobins are released, and hemolysis occurs. An artificial heart valve is in long-term contact with the bloodstream, so it is critical to reduce its damage to red blood cells. At the same time, artificial heart valve can cause the deformation of platelets and then coagulation due to the rupturing of blood 
Table 3

The result of hemolysis test for mastoid micro-structure PU surface with different periodic space

\begin{tabular}{llllcc}
\hline periodic space & \multicolumn{2}{l}{ testing value of OD } & \multicolumn{2}{c}{ Average } & $\begin{array}{c}\text { hemolysis } \\
\text { value } \\
\text { OD }\end{array}$ \\
\hline 0.25 & 1 & 2 & 3 & 0.037 & 2.49 \\
0.3 & 0.034 & 0.039 & 0.038 & 0.042 & 2.93 \\
0.43 & 0.045 & 0.040 & 0.041 & 0.024 & 1.33 \\
0.52 & 0.026 & 0.023 & 0.024 & 0.020 & 0.90 \\
Smooth & 0.019 & 0.022 & 0.021 & 0.057 & 4.26 \\
\hline
\end{tabular}

cells. By testing the numbers of erythrocyte hemoglobin released from the red blood cells after the tested material has been in contact with the blood for a certain time, the hemolysis rate of the material can be obtained to evaluate its hemolysis in vitro. Since hemolysis rate can sensitively, reliably and directly reflect the effect of exogenous biomaterials on red blood cells, it is a particularly important index for acute toxicity screening. When the anticoagulant properties of the material are studied, the extent of its damage on erythrocytes also must be considered. When red blood cells are lysed and hemoglobin is released, the concentration of the hemoglobin can be determined through the absorbance at $540 \mathrm{~nm}$.

The hemolysis ratio test results on the PU surfaces with different periodic space mastoid microstructures are shown in Table 3. The hemolysis ratio of all tested surfaces was less than 5\%, indicating that these samples are in line with the requirements of biological material hemolysis test. In addition, hemolysis ratio reduced with the increasing of the period space. When the periodic space was at maximum (0.52), the hemolysis ratio reached the lowest value, only $0.9 \%$. However for the mastoid micro-structure PU surface with period space of 0.3 , its hemolysis ratio was higher than that of the same material surface with period space of 0.25 , which was mainly due to the shape of the micro-structure. This testing result agreed with the results from previous dynamic coagulation experiments. Therefore, the generation of toxic substances was reduced by fabricating the proper micro-structure on the PU surface, and then its blood compatibility was improved.

\section{Conclusion}

$\mathrm{PU}$ is one of the biological materials commonly used in artificial heart valves. Micro-structures on the PU surface can help improve its hemocompatibility. The geometric parameters of the micro-structure affect the hemocompatibility. Generally, by increasing the periodic space of the micro-structure, the superhydrophobicity of the material is enhanced, and the number of platelet adhesions decreases. As a result, the dynamic clotting time is extended and the hemolysis ratio is reduced, which all contribute to improved hemocompatibility. In addition, the shape of the micro-structure also has a great impact on the hemo-compatibility of the PU. When the periodic space of the micro-structure on the PU surface is 0.25 , its hemocompatibility was superior to that of the PU surface with a periodic space of 0.3 . This could be caused by the suboptimal shape of the micro-structure. Collectively, both the geometric parameters and the shape of the micro-structure can 
affect the hemocompatibility. This finding can provide an intuitive guide for designing artificial heart valve surfaces in the future.

\section{Acknowledgement}

This research is supported by the Natural Science foundation of Jiangsu Province (BK2010203) and the Key Laboratory Opening foundation of Jiangsu Province (GZ201109) and Jiangsu Overseas Research \& Training Program for University Prominent Young \& Middle-aged Teachers and Presidents.

\section{References}

[1] J.M. Hung, L.H. Lin and Y.H. Shi, Research of microstructural characteristics on nanocrystalline diamond by microwave plasma CVD, Applied Surface Science 257 (2011), 5508-5512.

[2] R.J. Narayan, W. Wei, C. Jin et al., Microstructural and biological properties of nanocrystalline diamond coatings, Diamond \& Related Materials 15 (2006), 1935-1940.

[3] N. All, Y. Kousar, J. Gracio et al., Human microvascular endothelial cell seeding on Cr-DLC thin films for heart valve application, Thin Solid Films 515 (2006), 59-65.

[4] Y.X. Leng, J.Y. Chen, P. Yang et al., The microstructure and mechanical properties of $\mathrm{TiN}$ and $\mathrm{TiO}_{2} / \mathrm{TiN}$ duplex films synthesized by plasma immersion ion implantation and deposition on artificial heart valve, Surface \& Coatings Technology 201 (2006), 1012-1016.

[5] A. Bolz and M. Schaldach, Artificial heart valve: Improved blood compatibility by PECVD SiC: H coating, Artificial Organs 144 (1990), 260-269.

[6] J. Krzysztof and $\mathrm{K}$. Anna, The new generation $\mathrm{Ti}_{6} \mathrm{Al}_{4} \mathrm{~V}$ artificial heart valve with nanocrystalline diamond coating on the ring and with derlin disc after long-term mechanical fatigue examination, Diamond \& Related Materials 16 (2007), 1004-1009.

[7] J.M. Hung, C.Z. Wu and K.L. Ou, Researches of mechanical properties and biocompatibility on nanocrystalline diamond, Physics Procedia 32 (2012), 696-705.

[8] J.H. Zhang, H.B. Sun and G.M. Wang, Microstructural characterization of silicon-alloyed pyrolytic carbon for mechanical heart valves, Advanced Materials Research 230-232 (2011), 420-423.

[9] X. Ye, Y.L. Shao, M. Zhou et al., Research on micro-structure and hemo-compatibility of the artificial heart valve surface, Applied Surface Science 255 (2009), 6686-6690.

[10] Y.C. Jung and B. Bhushan, Mechanically durable carbon nanotube-composite hierarchical structures with superhydrophobicity, self-cleaning, and low-drag, ACS Nano. 3 (2009), 4155-4163.

[11] D. Ebert and B. Bhushan, Durable lotus-effect surfaces with hierarchical structure using micro-and nanosized hydrophobic silica particles, J. Collid. Interface Sci. 368 (2012), 584-591.

[12] L. Cao and D. Gao, Transparent superhydrophobic and highly oleophobic coatings, Faraday Discuss 146 (2010), 57-65.

[13] K.L. Cho, I.I. Liaw, AH-F. Wu and R.N. Lamb, Influence of roughness on a transparent superhydrophobic coating, J. Phys. Chem. C 114 (2010), 1228-1233.

[14] L. Xu, R.G. Karunakaran, J. Guo and S. Yang, Transparent, superhydrophobic surfaces from one-step spin coating of hydrophic nanoparticles, ACS Appl. Mater. Interfaces 4 (2012), 1118-1125.

[15] M. Im, H. Im, J.-H. Lee, J.-B. Yoon and Y.-K. Choi, A robust superhydrophobic surface with inverse-t rape-zoidal microstructures on a large transparent flexible substrate, Soft Matter 6 (2010), 1401-1404. 